\title{
"9+12 Gemeinsam gesund in Schwangerschaft und erstem Lebensjahr“
}

\author{
C. Wäscher ${ }^{1}$
}

\section{Zusammenfassung}

Das Projekt „9+12“ baut auf den Erkenntnissen der perinatalen Programmierung auf: Ein gesunder Lebensstil mit ausgewogener Ernährung und viel Bewegung in Schwangerschaft und erstem Lebensjahr wirkt präventiv in Bezug auf die Entwicklung von Übergewicht. Daher nutzt „9+12“ die Vorsorgeuntersuchungen bei Frauenärzten, Hebammen sowie Kinder- und Jugendärzten in diesem Zeitraum und erprobt im Landkreis Ludwigsburg einheitliche Beratungsinstrumente und Informationsmaterialien.

Stichworte: perinatale Programmierung, Vorsorgeuntersuchungen, Übergewicht, Schwangerschaft, Ernährung

\section{Hintergrund}

Die Weichen für ein gesundes Leben werden bereits in der Schwangerschaft und im ersten Lebensjahr gestellt. Nach aktuellem wissenschaftlichem Erkenntnisstand sind eine ausgewogene Ernährung und ausreichende Bewegung der Mutter beste Voraussetzungen für einen optimalen Start ins Leben $[1,13,20]$. Die medizinische Betreuung der (werdenden) Eltern und des heranwachsenden Kindes während dieser Lebensphase ist in unserem Gesundheitssystem fest verankert. Allerdings dienen die dafür genutzten Vorsorgeuntersuchungen bisher ausschließlich der Krankheitsfrüherkennung. Elemente der Übergewichtsprävention werden kaum berücksichtigt. Genau an dieser Stelle setzt das Modellprojekt „9+12 Gemeinsam gesund

1 Plattform Ernährung und Bewegung e.V. (peb)

Eingegangen: 12.03.2014

Angenommen durch Review: 19.04.2014 in Schwangerschaft und erstem Lebensjahr" der Plattform Ernährung und Bewegung e.V. (peb) an, nicht zuletzt, weil die Relevanz der Prävention von Übergewicht unter Wissenschaftlern unbestritten ist: Zunehmend leiden bereits Kinder und Jugendliche unter Übergewicht und Adipositas [18]. Laut deutschem Kinder- und Jugendgesundheitssurvey (KiGGS) sind $15 \%$ der jungen Menschen im Alter von 3-17 Jahren in Deutschland übergewichtig $[16,17]$.

\section{Metabolische Programmierung}

Das Ernährungs- und Bewegungsverhalten während der Schwangerschaft und im Säuglingsalter hat einen dauerhaften und prägenden Einfluss auf die spätere (Über-) Gewichtsentwicklung sowie auf das Risiko assoziierter Erkrankungen wie Diabetes mellitus Typ 2 und kardiovaskuläre Erkrankungen $[1,5,10,14]$. Bereits im Mutterleib wirken metabolische Faktoren auf den Fetus, die den Organismus somit langfristig beeinflussen [19]. Die Entwicklung des Kindes wird neben genetischen Faktoren auch durch externe Einflüsse wie mütterliche Ernährung, Hormone und Neurotransmitter bestimmt. Das Geburtsgewicht des Kindes wird unter anderem durch das Ernährungs- und Bewegungsverhalten sowie durch den Body-MassIndex (BMI) der Mutter beeinflusst. Sowohl ein hohes als auch ein niedriges Geburtsgewicht können das Risiko für späteres Übergewicht erhöhen [1,6]. Demzufolge gilt das Gesundheitsverhalten der Mutter als bedeutsame Determinante der prä- und postnatalen (vor- und nachgeburtlichen) Entwicklung des Kindes.

\section{$>$ Prä- und postnatale Bewegung der (werdenden) Mutter}

Körperliche Aktivität in der Schwangerschaft wirkt sich sowohl in psychosozialer als auch in physiologischer Hinsicht positiv auf Wohlbefinden und Gesundheit von Mutter und Kind aus. Besonders geeignet sind gemäß den Empfehlungen der Deutschen Gesellschaft für Sportmedizin \& Prävention (DGSP) 2011 folgende Sportarten [15]:

$>$ Radfahren in der Ebene (keine Gewichtsbelastung)

> Schwimmen, Wassergymnastik, Wasserfitness

> Wandern, Walking, Nordic Walking, Gymnastik, Jogging

$>$ sportliche Aktivität in der Höhe (diese sollte höhenangepasst erfolgen)

> dynamisches Krafttraining an Geräten (keine freien Gewichte) 
Sportlich aktive Frauen sind seltener von emotionalen Stimmungsschwankungen und postpartalen Depressionen (Wochenbett-Depressionen) betroffen [12,21]. Ausreichende Bewegung beeinflusst den Fettstoffwechsel positiv, senkt das Risiko für Gestationsdiabetes [13], wirkt einer starken Gewichtszunahme während der Schwangerschaft entgegen und erleichtert die Gewichtsnormalisierung nach der Geburt [4]. Darüber hinaus verbessern regelmäßige sportliche Aktivitäten die eigene Körperwahrnehmung und erhöhen die körperliche Leistungsfähigkeit. Dies sind Faktoren, die sich positiv auf den Geburtsvorgang auswirken und das Risiko für Geburtskomplikationen senken können. Die körperliche Fitness fördert außerdem den Heilungsprozess nach der Geburt. Frauen mit einem guten Fitnessstand können somit nach der Entbindung ihren aktiven Lebensstil schneller wieder aufnehmen $[12,14,15]$. Der optimale Bewegungsgrad richtet sich jedoch nach individueller Stilldauer und Konstitution vor und während der Schwangerschaft. Zu welchem Zeitpunkt eine Frau das volle Training wieder aufnehmen kann, hängt vom individuellen Fitnessstand ab und davon, ob die Frau ihr Kind stillt. Im Allgemeinen kann mit dem Training etwa 4 Wochen nach der Geburt begonnen werden. Im Vordergrund der ersten 6 Lebensmonate steht jedoch ein gezieltes Beckenbodentraining (Rückbildungsgymnastik) [15].

\section{$>$ Das 1. Lebensjahr}

Stillen senkt das Risiko für späteres Übergewicht und Adipositas. Studien zeigen, dass das Übergewichtsrisiko bei Kindern, die bis zum 5. Monat voll gestillt wurden, deutlich reduziert ist. Deshalb sollten Säuglinge mindestens bis zum Beginn des 5. Monats ausschließlich gestillt werden. Die Muttermilch deckt den Bedarf an Flüssigkeit und Nährstoffen des Kindes optimal, ist gut verdaulich, hygienisch einwandfrei, gut temperiert, enthält schützende Abwehrstoffe, ist jederzeit und überall verfügbar und kostengünstig. Darüber hinaus unterstützt Stillen die Bindung zwischen Mutter und Kind, fördert die Rückbildung der Gebärmutter und senkt das Risiko für Brust- und Eierstockkrebs [8].
Der durch das Stillen deutlich erhöhte mütterliche Energiebedarf (ca. 600 kcal pro Tag) unterstützt darüber hinaus nach der Geburt die Gewichtsnormalisierung der Mutter. Auch während der Stillzeit sollten sich Mütter den allgemeinen Empfehlungen entsprechend weiterhin ausgewogen ernähren. Von Diäten und Abnehmkuren ist in dieser Phase wegen des erhöhten Nährstoffbedarfs abzuraten [8].

Neben der Ernährung ist auch die Bewegungsförderung in Hinblick auf die Übergewichtsentstehung im Kindesalter relevant [3]. Gerade weil Kinder über einen sog. „natürlichen Bewegungsdrang“ verfügen, sollten Störfaktoren der gesunden Bewegungsentwicklung, wie z.B. die übermäßige Nutzung von Babywippen im 1. Lebensjahr, möglichst vermieden werden $[2,3,22]$.

\section{Modellprojekt}

„9+12 Gemeinsam gesund in Schwangerschaft und erstem Lebensjahr“"

Das Projekt „9+12 Gemeinsam gesund in Schwangerschaft und erstem Lebensjahr“ der Plattform Ernährung und Bewegung e.V. (peb) prüft, wie perinatale Übergewichtsprävention langfristig und wirkungsvoll in bereits vorhandenen Strukturen des Gesundheitssystems verankert werden kann. In der Modellregion Ludwigsburg werden angehende Eltern bereits mit der Feststellung der Schwangerschaft bis zum Ende des 1. Lebensjahres des Kindes bei der Umsetzung eines gesundheits-

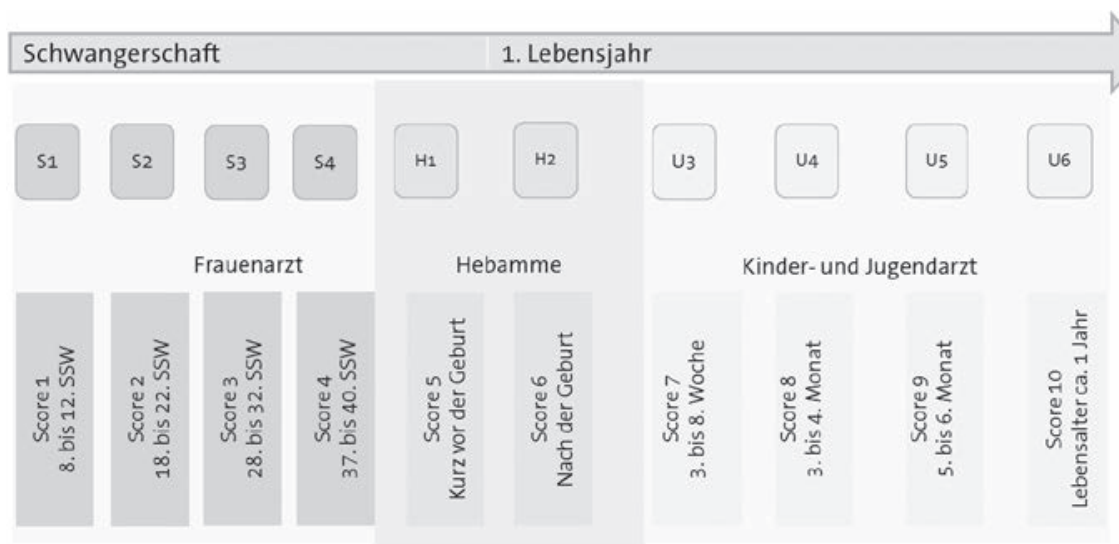

Abb. 1 Das Projekt „9+12“ nutzt insgesamt 10 Untersuchungszeitpunkte zur präventiven Beratung. @ Cornelia Wäscher sind. 1. Lebensjahres des Kindes (Abb.1). wickelt hat. förderlichen Lebensstils unterstützt. Einen guten Zugang zu der Zielgruppe bieten Frauenärzte, Hebammen sowie Kinderund Jugendärzte, weil sie wichtige Vertrauenspersonen und Ansprechpartner

Das Projekt verfolgt einen neuen Ansatz, in dem Vorsorgeuntersuchungen nicht mehr allein der Krankheitsfrüherkennung dienen, sondern darüber hinaus für systematische und ganzheitliche Beratungen zu den Themen Ernährung und Bewegung genutzt werden. Dazu nutzt das Projekt „9+12“ die 4 Schwangerschafts-Vorsorgeuntersuchungen beim Frauenarzt. Während und nach der Schwangerschaft werden die Hebammen mit 2 ergänzenden Interventionszeitpunkten in das Projekt eingebunden. Mit der Geburt folgen die Kinder- und Jugendärzte mit 4 weiteren Interventionszeitpunkten während des

Durch die Einbeziehung aller wichtigen medizinischen Akteure zielt das Modellprojekt „9+12“ darauf ab, interdisziplinäre Kooperationen zu stärken und somit die einheitliche Vermittlung qualitativer Botschaften sicherzustellen. Die peb kooperiert in dem Projekt eng mit dem aid-Netzwerk „Gesund ins Leben - Netzwerk Junge Familie“, welches einheitliche Handlungsempfehlungen für die Ernährung in der Schwangerschaft und im 1. Lebensjahr, in Abstimmung mit führenden Institutionen, Fachgesellschaften und Verbänden, ent-

Darüber hinaus hat peb zur Unterstützung der strukturierten Beratung gezielt Materialien entwickelt: Mit dem „Präventions- 
pass Ernährung und Bewegung“" werden wichtige Informationen zum Gesundheitsverhalten dokumentiert. Um einen Informationsabgleich zwischen den Beteiligten sicherzustellen, wird der Präventionspass in den Mutterpass sowie später in das Kindervorsorgeheft eingelegt. Mittels Fragebögen, den sog. „Präventions-Checklisten“, wird das Ernährungs- und Bewegungsverhalten erhoben, um eine bedarfsgerechte und motivierende Beratung zu gewährleisten.

Des Weiteren erhalten die Teilnehmerinnen Listen mit gesundheitsfördernden Angeboten rund um Schwangerschaft und Geburt im Landkreis Ludwigsburg. Neben Sportangeboten führen sie auch Ernährungs- und Laktationsberaterinnen und deren Kursangebote auf, damit (werdende) Mütter bei Bedarf ohne großen Aufwand die richtigen Ansprechpartner finden und die Empfehlungen der Ärzte und Hebammen umsetzen können.

Acht Kurzfilme (auf DVD), die auch auf dem YouTube-Kanal (gemeinsam gesund; s. Infokasten) verfügbar sind, ergänzen die persönliche Beratung durch die medizinischen Akteure. Die kurzen Sequenzen geben einfach umsetzbare Hinweise zu den Themen Ernährung und Bewegung. Darüber hinaus wurden in Zusammenarbeit mit den beteiligten Experten ein Flyer zum Bewegungsverhalten in der Schwangerschaft und eine Broschüre mit Bewegungstipps für das 1. Lebensjahr entwickelt.

Das Projekt startete im Januar 2012 und wird voraussichtlich bis Mitte 2014 in der Modellregion Ludwigsburg laufen. Um eine kontinuierliche Verbesserung der Projektabläufe zu gewährleisten und die Umsetzung in der Praxis zu erleichtern, bieten die Projektkoordinatoren regelmäßige Fortbildungen für die medizinischen Akteure an, beispielsweise zum Thema

\section{Weiterführende Informationen zum Modellprojekt „9+12“}

Projekt-Website: http://www.ernaehrung-und-bewegung.de/9plus12_das_ projekt.html

YouTube-Kanal "gemeinsamgesund“: http://www.youtube.com/user/ Gemeinsamgesund
„Ernährung und Bewegung in der Schwangerschaft“ oder „Gesprächsführung“. Derzeit nehmen rund 900 (werdende) Mütter am Projekt teil. In den kommenden Monaten sollen weitere schwangere Frauen für die Teilnahme am „9+12“-Projekt gewonnen werden. Im Oktober 2013 wurde „9+12“ mit dem Präventionspreis der Deutschen Adipositas-Gesellschaft (DAG) und deren Arbeitsgemeinschaft Adipositas im Kindes- und Jugendalter (AGA) ausgezeichnet.

\section{$>$ Wissenschaftliche Evaluation der Pilotphase}

Ein zentraler Teil des Projekts ist die wissenschaftliche Evaluation, um Erkenntnisse dazuzugewinnen, unter welchen Bedingungen es gelingt, präventive Maßnahmen in das bestehende Vorsorgesystem nachhaltig zu integrieren. Dabei sollen Schwierigkeiten und Hindernisse bei der Umsetzung des Projekts identifiziert und zugleich Lösungsansätze zur Optimierung entwickelt werden.

Erste Zwischenergebnisse der Evaluation, die in der 2. Hälfte des Jahres 2014 abgeschlossen wird, zeigen bereits überzeugende Projekterfolge. So wurden hinsichtlich der folgenden Aspekte signifikante Unterschiede zwischen der Modellregion (,9+12“-Intervention) und der Kontrollgruppe (keine Intervention) ermittelt:

$>$ Das Projekt erreicht überdurchschnittlich mehr adipöse Frauen (BMI >30) und mehr Frauen mit Migrationshintergrund.

> Schwangere erhalten in der Modellregion eher bewegungsförderliche Empfehlungen durch Frauenärzte und Hebammen und nehmen nach der Geburt eher an Rückbildungskursen teil bzw. planen dies als die Kontrollgruppe.

$>$ Auch ist in der Modellregion der Anteil der Frauen größer, die während der Schwangerschaft ihre alltäglichen Bewegungsaktivitäten verstärken. Schließlich fördern junge Mütter, die an der Intervention teilnehmen, eher das Bewegungsverhalten ihrer Kinder (z.B. durch Krabbeldecken) bzw. hemmen dieses seltener (durch Tragen des Kindes zuhause oder die Nutzung von Schaukelliegen).

\section{$>$ Ausblick}

Die bislang vorliegenden Ergebnisse der Evaluation weisen darauf hin, dass die im Projekt angewandten Maßnahmen zur Übergewichtprävention von den Teilnehmerinnen angenommen, umgesetzt und positiv bewertet werden. Zum jetzigen Zeitpunkt können jedoch noch keine Aussagen zur langfristigen Wirkung auf die Gewichtsentwicklung der Kinder getroffen werden. Um den nachhaltigen Erfolg der präventiven Beratungselemente zu prüfen, setzt sich die Plattform Ernährung und Bewegung e.V. dafür ein, dass nach Abschluss der 1. Projektphase eine Longitudinalstudie mit Follow-up-Untersuchungen in der Modellregion durchgeführt wird.

Um das Projekt in Ludwigsburg zu verstetigen, wird angestrebt, dass die bereits geschulten medizinischen Akteure nach einer Phase der Anpassung und Optimierung die präventiven Beratungen zu den Themen Ernährung und Bewegung auch nach Abschluss des Projekts weiterhin in ihren Praxen anbieten. Darüber hinaus ist eine Ausweitung des Projekts auf andere Regionen angedacht. Das übergeordnete Ziel ist, die gewonnenen Erkenntnisse für eine langfristige Implementierung der Projektmaßnahmen zur Übergewichtsprävention in das Gesundheitssystem zu integrieren.

Das Projekt „9+12“ wird aufgrund eines Beschlusses des Deutschen Bundestags durch „IN FORM - Deutschlands Initiative für gesunde Ernährung und mehr Bewegung des Bundesministeriums für Ernährung, Landwirtschaft und Verbraucherschutz“ gefördert.

Online zu finden unter

http://dx.doi.org/

10-1055/s-0034-1373869

\section{Literatur}

1 Brands B, Koletzko B. Frühe Ernährung und langfristiges Adipositasrisiko. Monatsschr Kinderheilkd 2012; 160: 1096-1102

2 Forschungsinstitut für Kinderernährung Dortmund. Der Ernährungsplan für das 
1. Lebensjahr. Im Internet: http://www. fke-do.de/index. php? module=page navigation\&index[page_navigation, action]=details\&index[page_navigation, data, page_navigation_id] $=62$; Stand: 28.10.2013

3 Graf C, Dordel S, Koch B et al. Bewegungsmangel und Übergewicht bei Kindern und Jugendlichen. Dtsch Z Sportmed 2006; 57: 220-225

4 Haakstad L, Voldner N, Henriksen T et al. Physical activity level and weight gain in a cohort of pregnant Norwegian women. Acta Obstet Gynecol Scand 2007; 86: 559-564

5 Harder T, Rodekamp E, Schellong K et al. Adipositas und perinatale Programmierung. In: Plagemann A, Dudenhausen J, Hrsg. Adipositas als Risiko in der Perinatalmedizin. München: Urban \& Vogel; 2010: 72-81

6 Harder T, Rodekamp E, Schellong K et al. Birth weight and subsequent risk of type 2 diabetes: a meta-analysis. Am J Epidemiol 2007; 165: 849-857

7 Koletzko B, Bauer C, Bung P et al. Ernährung in der Schwangerschaft - Teil 1. Dtsch med Wochenschr 2012; 137: 1309-1314

8 Koletzko B, Brönstrup A, Cremer M et al. Säuglingsernährung und Ernährung der stillenden Mutter. Monatsschr Kinderheilkd 2010; 158: 679-689

9 Koletzko B, Cetin I, Brenna J. Dietary fat intakes for pregnant and lactating women. Br J Nutr 2007; 98: 873-877

10 Koletzko B, Grote V, Schiess S et al. Prävention der kindlichen Adipositas durch die Säuglingsernährung. Monatsschr Kinderheilkd 2010; 158: 553-563

11 Koletzko B, Schiess S, Brands B et al. Frühkindliche Ernährung und späteres Adipositasrisiko. Bundesgesundheitsbl 2010; 53: 666-673

12 Korsten-Reck U, Marquardt K, Wurster KG. Schwangerschaft und Sport. Dtsch Z Sportmed 2009; 60: 117-121
13 Korsten-Reck U. Bewegung in Schwangerschaft und Stillperiode bei mütterlichem Übergewicht. Z Geburtshilfe Neonatol 2010; 214: 95-102

14 Korsten-Reck U. Bewegung in Schwangerschaft und Stillperiode bei mütterlichem Übergewicht. In: Plagemann A, Dudenhausen J, Hrsg. Adipositas als Risiko in der Perinatalmedizin. München: Urban \& Vogel; 2010: 102-118

15 Korsten-Reck U. Schwangerschaft und Sport. Teil 2: Praktische Empfehlungen für sporttreibende Frauen in Schwangerschaft und Wochenbett. Gynäkologe 2011; 44: 929-936

16 Kurth B, Schaffrath RA. Die Verbreitung von Übergewicht und Adipositas bei Kindern und Jugendlichen in Deutschland. Bundesgesundheitsbl 2007; 50: 736-743

17 Kurth B, Schaffrath RA. Übergewicht und Adipositas bei Kindern und Jugendlichen in Deutschland. Bundesgesundheitsbl 2010; 53: 643-652

18 Lobstein T. The prevention of obesity in children. Pediatr Endocrinol Rev 2004; 1 Suppl 3: 471-475

19 Schellong K, Rodekamp E, Harder T et al. Geburtsgewicht, Gestationsdiabetes und Perinatale Programmierung. Gynakol Geburtsmed Gynakol Endokrinol 2009; 5: 182-192

\section{Summary}

\section{„9+12: Healthy during pregnancy and first year of life“}

The project „ $9+12$ “ builds on the findings of the perinatal programming: „A healthy lifestyle with a balanced diet and plenty of exercise in pregnancy and the first year of life leads to the prevention of obesity development“. Thus „9+12“ uses the preventative medical checkups by gynecologists, midwives, as well as pediatricians and tests the joint advisory tools and information materials in the District of Ludwigsburg.

Key words: perinatal programming, preventive medical checkups, obesity 